\title{
Pilot Study of Sentinel-Node-Based Adoptive Immunotherapy in Advanced Colorectal Cancer
}

\author{
Mona Karlsson, $\mathrm{PhD}^{1}$, Per Marits, $\mathrm{PhD}^{1}$, Kjell Dahl, $\mathrm{PhD}^{2}$, Tobias Dagöö, $\mathrm{MD}^{3}$, Sven Enerbäck ${ }^{4}$, Magnus Thörn ${ }^{2}$, \\ and Ola Winquist ${ }^{1}$
}

${ }^{1}$ Karolinska Institute, Department of Medicine, Unit of Clinical Allergy Research, Karolinska University Hospital, Stockholm, Sweden; ${ }^{2}$ Karolinska Institute, Department of Surgery, South Stockholm General Hospital, Stockholm, Sweden; ${ }^{3}$ Karolinska Institute, Department of Radiology, South Stockholm General Hospital, Stockholm, Sweden; ${ }^{4}$ The Sahlgrenska Academy, Institute of Medical Biochemistry, Göteborg University, Gothenburg, Sweden

\begin{abstract}
Background. Despite optimal surgical treatment and modern adjuvant therapies, $50 \%$ of patients diagnosed with colorectal cancer die within 5 years. Immunotherapy offers an appealing complement to traditional chemotherapy, with possible long-term protection against tumor recurrences through immunological memory. We have conducted a pilot study of a novel adoptive immunotherapy, using autologous, in vitro expanded lymphocytes isolated from the tumor-draining sentinel lymph node.

Study Design. Sentinel nodes were recovered from 16 patients with disseminated or locally advanced, high-risk colorectal cancer. Single-cell suspensions of sentinel-nodeacquired lymphocytes were clonally expanded in vitro in the presence of autologous tumor extract and returned as a transfusion. Patients were followed with clinical and radiological evaluations. Long-term survival was compared with traditionally treated controls.

Results. Sentinel-node-acquired $\mathrm{CD}^{+}{ }^{+}$Th1-lymphocytes could be clonally expanded in vitro and safely administered to all 16 patients without side-effects. In four out of nine stage IV patients, complete tumor regression occurred. Median survival time in the stage IV patients $(n=9)$ was
\end{abstract}

Per Marits and Kjell Dahl contributed equally to this work.

(C) The Author(s) 2010. This article is published with open access at Springerlink.com

First Received: 8 September 2008;

Published Online: 30 January 2010

M. Thörn

e-mail: magnus.thorn@sodersjukhuset.se

O. Winqvist

e-mail: ola.winqvist@karolinska.se
2.6 years, as compared with 0.8 years in conventionally treated controls. A dose-dependent effect with regards to reduced tumor burden and long-term survival was observed. Conclusion. Sentinel-node-based adoptive immunotherapy is feasible; the method has shown no apparent sideeffects and appears to convey therapeutic antitumor effects. Further studies are justified to determine its efficacy and precise role in the treatment of colorectal cancer.

Colorectal cancer is the third most common cause of cancer mortality. ${ }^{1}$ Despite surgery and adjuvant therapies, half of patients have recurrences and ultimately die within 5 years from diagnosis. ${ }^{2}$ Patients with locoregional lymph node metastases (stage III) have an approximately 60\% 5year survival after surgery and adjuvant therapy, but when distant metastases are present the 5-year survival rate is less than $10 \%{ }^{3}$ The established adjuvant treatment in stage III patients was 5-fluorouracil (5-FU)-based chemotherapy at the time of the study, which has been reported to improve absolute 5 -year survival at most by $10 \%{ }^{4,5}$ In stage II patients the statistically significant, absolute improvement in survival with chemotherapy is even smaller, and it is not generally recommended to all stage II patients. ${ }^{6}$ However, $20 \%$ of patients who are classified as being lymph node negative will suffer from recurrent disease. $^{3}$ Attempts have been made to identify tumor characteristics conferring high risk of recurrence in order to offer adjuvant treatment exclusively to stage II patients with such tumors. Still, it is not clear whether this strategy is successful, and no consensus exists on which tumor characteristics are the most useful to predict relapse.

The most important negative prognostic factor for survival of colorectal cancer is the detection of lymph node or 
distant metastases, which are present in about $50 \%$ of all cases. ${ }^{7}$ Understaging is likely, since it is widely accepted that accurate staging of colon cancer is difficult and critically dependent on the number of lymph nodes analyzed by the pathologist. ${ }^{8}$ The sentinel node, originally defined in penile carcinoma, is the first lymph node to receive lymphatic drainage from a tumor. ${ }^{9}$ Sentinel node detection and analysis have recently been applied in colon cancer, improving staging considerably. ${ }^{10,11}$ To test if T-lymphocytes were components of a natural, tumor-evoked immune response originating in tumor-draining lymph nodes, we analyzed sentinel nodes from patients with urinary bladder cancer and colon cancer. ${ }^{12-14}$ In those nodes we identified tumor-reactive lymphocytes, secreting interferon (IFN)- $\gamma$ upon stimulation with tumor antigen in addition to dosedependent proliferation, when assessed ex vivo directly after dissection. The sentinel node was the predominant location for activation and expansion of tumor-reactive lymphocytes.

Vaccination trials have shown promising results in colorectal cancer patients. ${ }^{15-17}$ Adoptive immunotherapy, including collection and expansion of autologous tumorreactive lymphocytes with retransfusion to the patient, has been explored in malignant melanoma, and recently a retroviral gene therapy approach using a melanoma-specific T-cell receptor has been tried. ${ }^{18,19}$ Immunotherapy offers an appealing complement to traditional chemotherapy, with possible long-term protection against tumor recurrences through immunological memory. Hitherto, the majority of studies have used peripheral blood or tumorinfiltrating lymphocytes as the source of $\mathrm{CD}^{+}$cytotoxic $\mathrm{T}$ lymphocytes for activation and expansion.

We became interested in the specific $\beta$-cell destruction observed in a mouse model, after transfer of in vitro $\beta$-cell surrogate antigen-activated $\mathrm{CD} 4^{+}$Th1-cells. ${ }^{20}$ In another mouse model, presence of $\mathrm{CD}^{+}$T-helper cells appeared necessary for the effective functioning of cytotoxic $\mathrm{T}$ lymphocytes and for the development of memory cells. ${ }^{21}$ Furthermore, $\mathrm{CD}^{+}$T-helper-1 cells mature due to the expression of the transcription factor T-bet, which also induces production of IFN- $\gamma$ necessary for effective defense against metastases. ${ }^{22,23}$ We reasoned that tumoractivated $\mathrm{CD}^{+}$Th1-cells harvested from the natural source, the tumor-draining sentinel node, may after in vitro expansion elicit a similar response in patients with disseminated cancer, eradicating metastatic cells which express antigens derived from the tumor. In the present study we obtained sentinel nodes from patients with disseminated or locally advanced, high-risk colon cancer with aggressive tumor characteristics such as ulcerations, vascular or perineural invasion. Sentinel or metinel (i.e., metastasis-draining lymph node) node-acquired lymphocytes were expanded ex vivo by stimulation with autologous tumor extract and retransfused into the patient with the primary objective of investigating feasibility, toxicity, and side-effects and secondarily to evaluate its effect on long-term tumor progression. ${ }^{14}$

\section{METHODS}

\section{Study Design}

This was a pilot study, approved by the Local Ethical Committee at Karolinska Institute, Stockholm, Sweden. The inclusion criterion was operation due to metastatic or locally advanced, high-risk colon cancer, with peroperative sentinel node detection.

\section{Patients}

Sixteen consecutive patients diagnosed with colon cancer (six women and 10 men, average age 62 years) were included from September 2003 and followed with regular controls at the hospital until February 2008 (Table 1). Patients were informed both by surgeons and oncologists not participating in the study, and each patient gave informed consent. Patients were histopathologically classified as stage II, III or IV, as defined by American Joint Committee on Cancer (AJCC) criteria. All five patients with stage II disease were identified as high risk according to the vascular emboli, lymphatic invasion, and perineural invasion (VELIPI) criteria. ${ }^{24}$ They had aggressive tumor characteristics such as ulcerations, vascular or perineural invasion. Three patients (nos. 10, 13, and 15) had previously been treated for primary colon cancer and now had recurrent disease in the liver (nos. 10 and 13) or abdominally (no. 15). None of the patients had received any organ transplant, nor had a history of steroid treatment or other immunosuppressive regimes.

\section{Sentinel Node Detection}

Identification of sentinel nodes was performed intraoperatively using the sentinel node technique. ${ }^{10}$ Mobilization of the colonic tumor or metastasis was conducted according to standard surgical procedures. Patent blue dye (Guerbet, Paris) was injected superficially in the serosa around the tumor or in the tissue surrounding the metastasis. One to three-first draining lymph nodes were colored blue, usually within $5 \mathrm{~min}$. These sentinel nodes were marked with sutures and removed when the resection was complete (Fig. 1). Approximately half of each sentinel lymph node was retained for analysis and expansion, the 
TABLE 1 Participant characteristics

\begin{tabular}{|c|c|c|c|c|c|c|c|c|}
\hline Patient no. & Age/sex & $\begin{array}{l}\text { AJCC stage/TNM } \\
\text { classification } \\
\text { at surgery }\end{array}$ & $\begin{array}{l}\text { Infused cells } \\
\left(\times 10^{6}\right)\end{array}$ & $\mathrm{CD} / \mathrm{CD} 8^{\mathrm{a}}$ & $\mathrm{IFN}-\gamma(\mathrm{pg} / \mathrm{ml})$ & $\begin{array}{l}\text { IL-4 } \\
(\mathrm{pg} / \mathrm{ml})\end{array}$ & Response & $\begin{array}{l}\text { Overall } \\
\text { survival } \\
\text { (months) }\end{array}$ \\
\hline 1 & $66 / \mathrm{M}$ & IIA/T3N0M0 & 152 & $96 / 1.4$ & 1411 & 6 & SD & 42 \\
\hline 2 & $74 / \mathrm{M}$ & IIA/T3N0M0 & 63 & $64 / 22$ & ND & ND & $\mathrm{SD}^{\mathrm{b}}$ & 44 \\
\hline 3 & 71/M & IIA/T3N0M0 & 50 & $74 / 15$ & 2091 & 5 & SD & 44 \\
\hline 4 & $67 / \mathrm{F}$ & IIA/T3N0M0 & 8 & $15 / 51$ & ND & ND & SD & 45 \\
\hline 5 & $67 / \mathrm{M}$ & IIA/T3N0M0 & 4 & $92 / 0.2$ & ND & ND & SD & 46 \\
\hline 6 & $58 / \mathrm{F}$ & IIIA/T2N1M0 & 16 & $77 / 18$ & 417 & 9 & SD & 36 \\
\hline 7 & $64 / \mathrm{F}$ & IIIC/T3N2M0 & 113 & $64 / 25$ & ND & ND & SD & 49 \\
\hline 8 & $61 / \mathrm{F}$ & IV/T2N1M1 & 1 & $3.7 / 35$ & ND & ND & $\mathrm{SD}^{\mathrm{c}}$ & 6 \\
\hline 9 & $82 / \mathrm{F}$ & IV/T3N2M1 & 12 & $98 / 0.1$ & ND & ND & $-^{\mathrm{d}}$ & 6 \\
\hline 10 & $54 / \mathrm{M}$ & IV/T3N1M1 & 40 & $37 / 24$ & ND & ND & SD & 51 \\
\hline 11 & $33 / \mathrm{M}$ & IV/T4N2M1 & 40 & $87 / 3.3$ & 908 & 19 & $\mathrm{SD}^{\mathrm{e}}$ & 12 \\
\hline 12 & $66 / \mathrm{M}$ & IV/T3N1M1 & 40 & $46 / 27$ & 764 & 6 & $P R^{f}$ & 15 \\
\hline 13 & $74 / \mathrm{M}$ & IV/TXNXM1 & 170 & $73 / 22$ & 142 & 18 & $\mathrm{CR}^{\mathrm{g}}$ & 31 \\
\hline 14 & $42 / \mathrm{F}$ & IV/T4N0M1 & 80 & $66 / 11$ & ND & ND & $\mathrm{CR}^{\mathrm{h}}$ & 40 \\
\hline 15 & $47 / \mathrm{M}$ & IV/TXNXM1 & 80 & $24 / 16$ & ND & ND & $\mathrm{CR}$ & 51 \\
\hline 16 & $65 / \mathrm{M}$ & IV/T3N2MX & 270 & $82 / 15$ & ND & ND & $\mathrm{CR}^{\mathrm{i}}$ & 51 \\
\hline
\end{tabular}

TNM tumor-node-metastasis

Complete response (CR), partial response (PR), and stable disease (SD) as evaluated by WHO criteria at follow-up

a The numbers represent the percentage of CD4- and CD8-positive cells detected with FACS

b The patient received lymphocyte transfusion 1 month after surgery, when he displayed stable disease for 6 months. Due to the appearance of liver metastases after 6 months, he was treated with chemotherapy and later liver surgery. The patient developed recurrent disease after another 30 months

${ }^{c}$ Patient 8 displayed regression of liver metastases and decreased CEA levels, but radiological evaluation did not fulfill the WHO criteria for partial response and the patient was classified as having stable disease. The patient's physical condition improved following immunotherapy, with regain of appetite and weight, and from being bedridden to daily walks and kayaking (WHO class I). At this time she suddenly died at home. Relatives opposed the performance of autopsy, but it is likely that heart attack or lung embolus was the cause of death

d The patient was an 82-year-old lady with cardiopulmonary disease, known and treated prior to her development of colon cancer. At the time of diagnosis of colon cancer she had metastases in liver and spleen, and bilateral lung metastases. After immunotherapy she lived in a geriatric ward and died 5 months later. No autopsy was done. Lost to follow-up

e The patient was a young man who presented as an emergency with colonic obstruction and metastatic disease (intraperitoneal and liver). The primary tumor causing the obstruction was resected. The tumor responded initially, with regression of liver and peritoneal metastases. In addition, ascites disappeared with decreased S-CEA levels and enhanced general well-being. After 9 months the disease progressed and he died 3 months later

${ }^{\mathrm{f}}$ After immunotherapy the patient achieved partial regression of metastases to the liver and lungs. After 7 months he developed a metastasis to the vertebral column. One month later the lung metastases increased in size and after further 4 months the liver metastases increased in size and number. He died 14 months after the immunotherapy

g A 74-year old man who initially had surgery due to a sigmoid colon cancer, stage II. Fifteen months later he developed a solitary liver metastasis. Following liver surgery he had adjuvant sentinel-node-based immunotherapy. CEA was stable and he showed no signs of recurrence. One month later he developed pancreatic cancer and died after a further 13 months

h The patient displayed complete response after immunotherapy; relapse occurred after approximately 3 years, with metastases to the liver, and after 40 months the patient died from her disease

i The patient had multiple lymph node metastases $(n=16)$ and presented immediately after primary surgery with liver metastasis. Following incomplete resection of the liver metastasis, he received sentinel-node-based immunotherapy, which resulted in complete response of remaining metastases. Two years later he developed additional liver metastases and received further immunotherapy based on expansion of peripheral blood lymphocytes using autologous tumor extract

other half being processed for routine histopathological examination by hematoxylin and eosin. A piece of the tumor or metastasis, including the invasive margin, was used for antigen preparation.

\section{Cell Culture}

The sentinel node material was retained on ice and thereafter in AIM $\mathrm{V}^{\circledR}$ media (Invitrogen) in all subsequent 

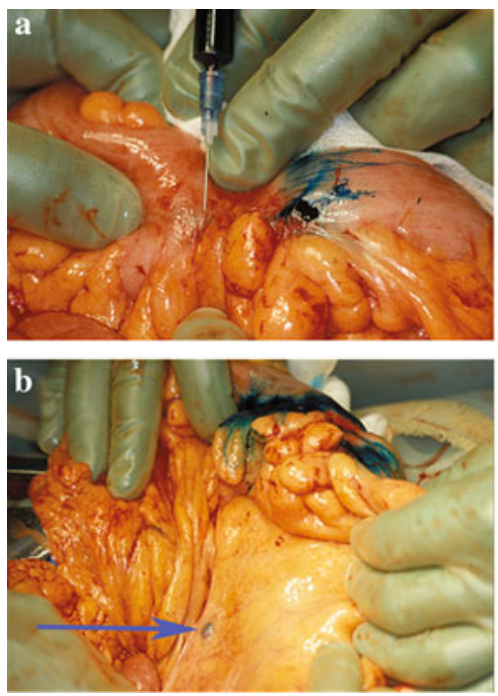

c
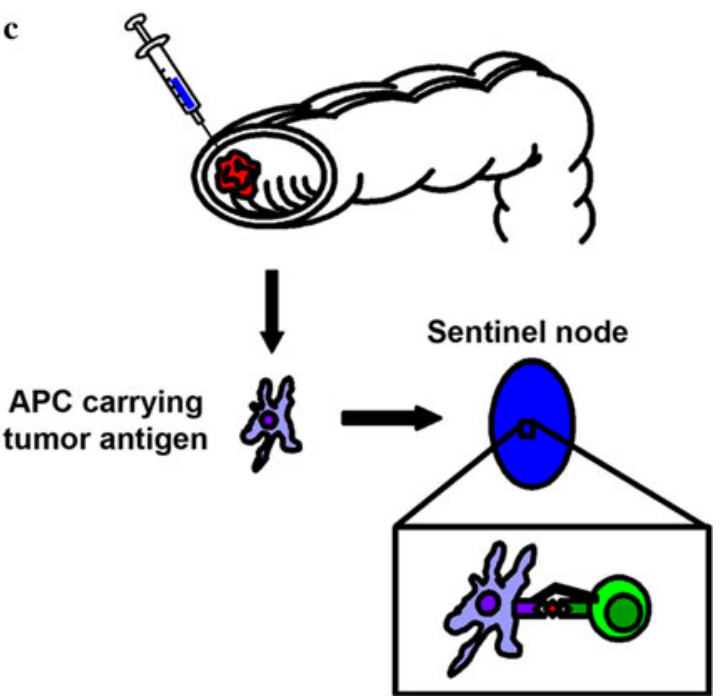

FIG. 1 Perioperative identification of sentinel node(s). Identification of sentinel nodes was performed by subserosal injections of Patent blue dye at four sites around the tumor (a). Usually within $5 \mathrm{~min}$, one or more blue-colored lymph nodes appear in the mesentery (b). Hypothetical scheme of activation of tumor-reactive lymphocytes in the sentinel node (c). In the tumor there is a rapid turnover of cells,

steps. Single-cell suspensions of sentinel node lymphocytes were obtained through gentle homogenization in a loose-fit glass homogenizer, following which cells were washed twice in medium. The sentinel node lymphocytes were dispersed into cell culture flasks at a density of 4 million cells/ml and supplemented with interleukin-2 (IL-2) (Proleukin ${ }^{\circledR}$, Chiron). Autologous tumor extract was prepared by homogenization with an Ultra Turrax in 5 volumes (w/ v) of $2 \times$ phosphate-buffered saline (PBS) followed by denaturation for $5 \mathrm{~min}$ at $97^{\circ} \mathrm{C}$. Three to four days after initiation of the cell culture, autologous tumor extract was added at dilution of $1 / 100(\mathrm{v} / \mathrm{v})$. For long-term culture the cells were retained in a cell incubator at $37^{\circ} \mathrm{C}$ and $5 \% \mathrm{CO}_{2}$, and IL-2 (Proleukin ${ }^{\circledR}$, Chiron) was added every 3-4 days. After approximately 3 weeks the cell cultures were restimulated through the addition of autologous tumor extract at dilution of $1 / 100(\mathrm{v} / \mathrm{v})$. For efficient antigen presentation, autologous peripheral blood mononuclear cells (PBMC)were collected using Ficoll-Paque PLUS (Amersham Biosciences, GE Healthcare), irradiated with 2,500 rad, and added to the cell cultures. The day before transfusion, viable cells from the cultures were retrieved using Ficoll-Paque PLUS (Amersham Biosciences, GE Healthcare). On the day of transfusion, the cells were washed twice in saline solution (sodium chloride Baxter Viaflo $9 \mathrm{mg} / \mathrm{ml}$, Baxter) and then transferred to a sterile plastic bag containing 100-200 ml saline solution and 1\% human serum albumin (Baxter). Investigations to exclude and lack of oxygen and nutrition, causing a hostile environment which attracts macrophages and dendritic cells. These professional antigenpresenting cells (APC) phagocytose debris from tumor cells and then migrate via the lymph vessels to the draining sentinel node. In the sentinel node the APCs present tumor-derived peptides, thus activating tumor-reactive T-lymphocytes

microbial growth were performed prior to transfusion. Infusion of the cells back into the patient was performed according to the blood transfusion guidelines of the hospital.

\section{Immunological Evaluation}

The proliferative response upon stimulation with autologous tumor homogenate in PBMC was investigated using a tritium-labeled thymidine incorporation proliferation assay. PBMC were obtained by density centrifugation (Ficoll-Paque, Amersham Biosciences, GE Healthcare). Cells were resuspended and washed twice in Roswell Park Memorial Institute (RPMI) 1640 (Life Technologies) containing $2.5 \%$ fetal calf serum (FCS) (Life Technologies) before culture in RPMI 1640 medium containing 10\% human AB serum (Sigma), 1\% penicillin-streptomycin (Sigma), and $1 \%$ glutamine (Sigma). Lymphocytes were incubated at $3 \times 10^{5}$ cells/well in a 96-well plate and stimulated with tumor homogenate diluted $1 / 100$ or $1 / 10$ (v/v) or with the general T-lymphocyte mitogen Concanavalin A (Con A, $10 \mu \mathrm{g} / \mathrm{ml}$; Sigma) in triplicate. Proliferation was measured on day 2 (Con A) and days 5, 6, and 7 (tumor homogenate) by adding $1 \mu \mathrm{Ci}^{3} \mathrm{H}$-thymidine/ well (Amersham) $18 \mathrm{~h}$ prior to harvesting. Samples were subjected to scintillation counting. Stimulation of PBMC for measurement of IFN- $\gamma$ secretion was performed in 96well plates with $3 \times 10^{5}$ cells/well in triplicate with tumor 
homogenate diluted $1 / 10$ and $1 / 100(\mathrm{v} / \mathrm{v})$, or Con A $(10 \mu \mathrm{g} /$ $\mathrm{ml}$, Sigma). The amount of secreted IFN- $\gamma$ was measured by enzyme-linked immunosorbent assay (ELISA, Human IFN- $\gamma$ Duoset; R\&D Systems) on culture supernatants in pooled triplicate samples. At the end of ex vivo culture of sentinel-node-acquired lymphocytes the amount of IFN- $\gamma$ and IL-4 secreted into the supernatant was measured in triplicate by ELISA (Human IFN- $\gamma$ Duoset and Human IL4 Duoset, R\&D Systems).

\section{Flow Cytometry Analyses}

Cells from the sentinel node, nonsentinel node, peripheral blood leukocytes (PBL), and from the tumor itself were characterized by fluorescence-activated cell sorting (FACS). Samples were analyzed by FACS regularly from the cultured sentinel-node-acquired lymphocytes. Cells were incubated for $30 \mathrm{~min}$ in PBS supplemented with $2 \%$ FCS and $0.05 \% \mathrm{NaN}_{3}$ (FACS buffer) with antibodies against markers for immune cell subpopulations and for lymphocyte activation. Antibodies conjugated with fluorescein isothiocyanate (FITC) specific for the following markers were used: CD69, HLA-DR, CD45RA, CD25; conjugated with phycoerythrin (PE): CD62L, CD19, CD45RO, CD56, conjugated with peridinin-chlorophyllprotein (PerCP): CD8, CD3; conjugated with allophycocyanin (APC): CD4, CD14, CD8. The V $\beta$-TCR repertoire was examined using the Beta mark kit (Beckman Coulter), $5 \times 10^{5}$ cells/tube being stained in $10 \mu$ of the eight different vials containing mixtures of FITC, PE, and dualcolor FITC-PE-conjugated TCR V $\beta$ antibodies. In addition, direct conjugated antibodies CD8 PerCP and CD4 APC were added to each tube. The presence of tumor cells was examined by intracellular staining for cytokeratin 20. Cells were permeabilized in FACS buffer containing $0.3 \%$ saponin, incubated for $30 \mathrm{~min}$ with anti-human cytokeratin 20 (Dako Cytomation), washed, and stained with FITCconjugated donkey anti-mouse immunoglobulin $\mathrm{G}$ (IgG) for 30 min (Jackson ImmunoResearch).

\section{Chemotherapy}

Six patients (nos. 1, 3-5, 8, and 9) did not receive any chemotherapy. Patients 13 and 15 received chemotherapy after primary surgery. They later developed metastases (at least 1 year after chemotherapy) and at that time had sentinel-node-based immunotherapy, without any further chemotherapy. Patients $2,6,7,10-12,14$, and 16 were treated with immunotherapy followed by chemotherapy (first-line 5-fluorouracil + leucovorin). Adverse effects related to chemotherapy and limited patient compliance led to early termination of chemotherapy treatment in four patients (nos. 6, 10, 12, and 14). Two stage IV patients received no chemotherapy treatment (nos. 8 and 9), and two patients received chemotherapy 1 year prior to immunotherapy (nos. 13 and 15).

\section{Follow-up}

Patients were clinically evaluated according to the regular follow-up program after diagnosis and treatment of colon cancer in Sweden. Follow-up visits usually took place $3,6,12$, and 18 months after cell transfusion. On each of these occasions, general condition was estimated and the amount of carcinoembryonic antigen (CEA) in serum was measured. Every 6 months the patients were also examined for presence of liver and lung metastases by ultrasound, X-ray or computer tomography (CT) scan, or fluorodeoxyglucose positron emission tomography (FDGPET). Assessment of clinical response was made by comparison of radiographic measurements and physical examinations before and after treatment using World Health Organization (WHO) criteria. Complete disappearance of all evaluable disease was defined as complete response (CR). Partial response (PR) was defined as decrease of $50 \%$ or more in the sum of the products of perpendicular diameters of all measurable lesions. Stable disease (SD) was defined as no signs of tumor progression between two consecutive CT evaluations of measurable remaining disease or lack of additional (stage IV) or no appearance of metastatic lesions (stage II and III).

\section{Statistical Analysis}

The number of cells received by patients with SD and PR disease was compared with the number of cells received by patients classified as CR using Wilcoxon's rank-sum test. All patients in the Stockholm colon cancer registry diagnosed during the year 2003 with stage IV ( $n=174)$ were used as a control group for cumulative survival comparison. The survival time of the nine stage IV patients treated with immunotherapy and the control stage IV cohort were plotted in an actuarial survival diagram. Survival times were compared using log-rank (MantelCox) analysis. $P<0.05$ was considered significant.

\section{RESULTS}

\section{Expansion of Sentinel-Node-Acquired Lymphocytes}

Sixteen patients with colorectal cancer were studied (Table 1). In all patients, 1-3 (average 2.1) sentinel node(s) were identified intraoperatively (Fig. 1) by peritumoral injections of Patent blue dye (Guerbet, Paris). We 
a

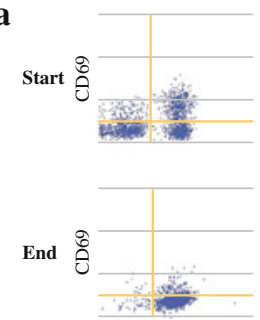

CD4
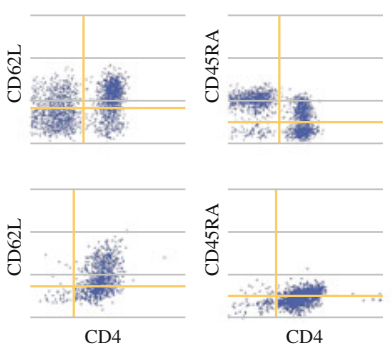

$\mathrm{CD} 4$
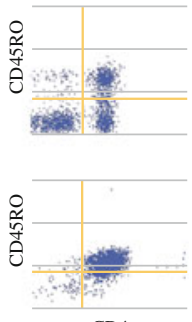

CD4

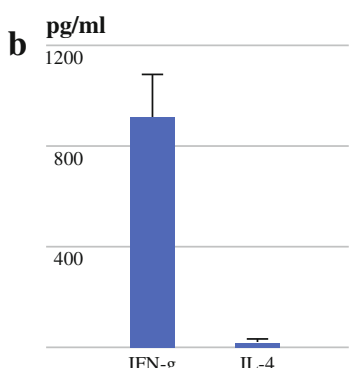

$\%$ of $\mathrm{CD4}^{+} \mathrm{T}$

c lymphocytes

70 Sentinel node lymphocyte

End of ex vivo culturing

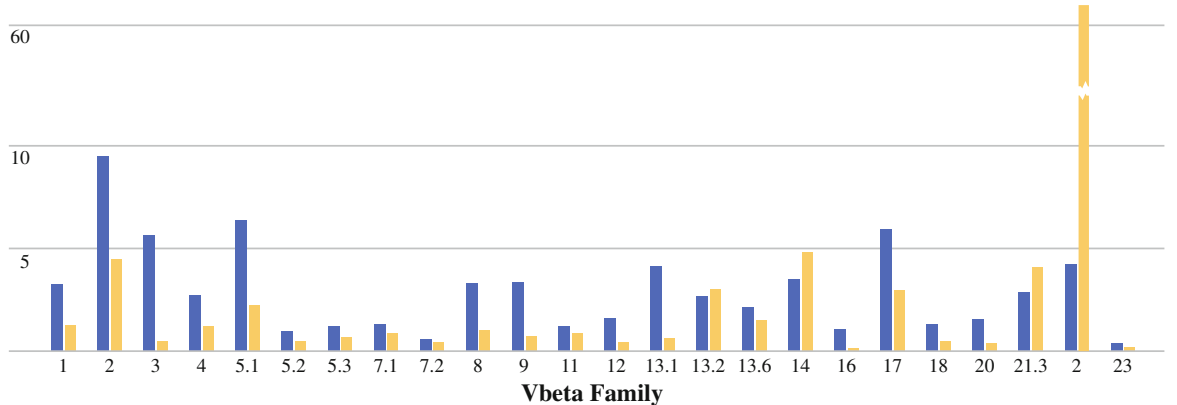

FIG. 2 Sentinel node lymphocytes are enriched in T-helper cells and secrete IFN- $\gamma$ after ex vivo cell culturing. At the start and end of ex vivo cell culture, sentinel-node-acquired lymphocytes were characterized by flow cytometry with regards to activation and memory cell markers (a). The amount of IFN- $\gamma$ and IL-4 secreted into the supernatant was measured by ELISA (b). The data presented is from patient 15 on the day prior to transfusion, which in this case corresponds to 40 days of ex vivo cell culture. T-cell receptor $\mathrm{V} \beta$ repertoire of expanded sentinel-node-acquired lymphocytes (c). In patient 7 the $\mathrm{T}$-cell receptor $\mathrm{V} \beta$ repertoire was investigated by flow cytometry at the start and end of ex vivo cell culturing. Clonal $(\mathrm{V} \beta$ 22) expansion of $\mathrm{CD} 4^{+} \mathrm{T}$-helper lymphocytes was detected cultivated sentinel-node-acquired lymphocytes by stimulation with autologous tumor extract, to ensure clonal expansion of tumor-reactive $\mathrm{CD} 4^{+}$Th1-lymphocytes. Low amounts of interleukin-2 were added to overcome immunosuppression. The average number of sentinel-nodeacquired lymphocytes at the start of expansion was 115.2 million cells (range 3.6-509 million, median 71 million). Cells were continuously monitored and characterized by flow cytometry (FACS) during expansion (Fig. 2a). The $\mathrm{CD} 4^{+} / \mathrm{CD}^{+}$ratio at the start was 4.8 on average (range 0.36-10, median 5.0), indicating a natural enrichment of $\mathrm{CD} 4{ }^{+}$T-helper lymphocytes in sentinel nodes when compared with the CD4/CD8 ratio in peripheral blood (range 1.0-2.5). In addition, B-lymphocytes $\left(\mathrm{CD} 19^{+}\right)$and a small number of natural killer (NK) cells $\left(\mathrm{CD}^{2} 6^{+}, \mathrm{CD}^{-}\right)$were present in sentinel nodes (not shown). The cells were held in culture for 36.5 days on average (range 23-58 days). Initially, the total number of cells decreased. B-lymphocytes disappeared almost completely $(37.6 \%$ at start, $0.4 \%$ at transfusion), and the number of $\mathrm{CD}^{+}{ }^{+} \mathrm{T}$-cytotoxic cells diminished. This procedure mainly promoted the expansion of T-helper lymphocytes, since the average CD4/CD8 ratio at transfusion was 86.6 (Fig. 2a, Table 1). However, in some cases (Table 1) a substantial number of $\mathrm{CD} 8^{+} \mathrm{T}$ cytotoxic lymphocytes developed. The transfused cells were heterogenous with respect to CD62L expression, but uniformly CD45RO positive, indicative of a mixed memory-cell population, comprising both central and effector memory cells (Fig. 2a).

\section{ELISA Results}

In six cases (Table 1), the expanded T-lymphocytes were functionally tested before transfusion by measuring the levels of the Th1 cytokine IFN- $\gamma$ and the Th2 cytokine IL-4 in the cell culture supernatants (Fig. 2b). The average level of IFN- $\gamma$ was $956 \mathrm{pg} / \mathrm{ml}$ and the average level of IL-4 was $11 \mathrm{pg} / \mathrm{ml}$, indicating that the expanded T- lymphocytes were functional and Th1 responsive. Restimulation after about 3 weeks with autologous tumor antigen and autologous PBMCs, acting as antigen-presenting cells, resulted in further clonal expansion of tumor-reactive T-lymphocytes as assessed by investigating the $\mathrm{T}$-cell receptor $\mathrm{V} \beta$ repertoire of sentinel-node-acquired lymphocytes before and after restimulation (Fig. 2c). 

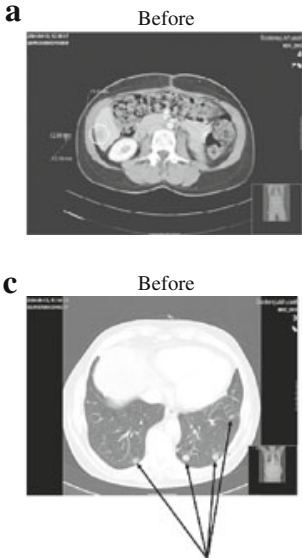
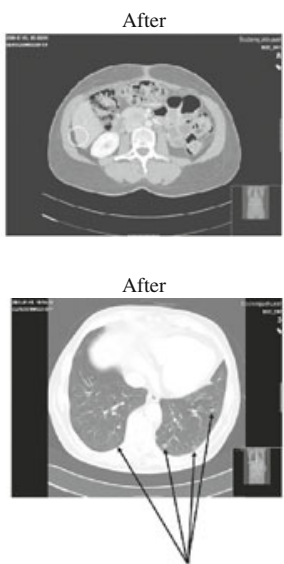

FIG. 3 Patient outcome. Computer tomography (CT) of patient 14, showing a metastasis measuring $12.7 \times 13.0 \times 13.3 \mathrm{~mm}^{3}$ in the liver at time of treatment (a, left panel). Follow-up CT scan 3 months after treatment showed no signs of liver metastasis. (a, right panel). FDGPET of patient 14 at time of treatment revealed additional FDGpositive metastases in the liver, indicated by arrows (b, left panel). Follow-up FDG-PET scan 6 months after treatment without any signs of FDG-positive metastases, indicating complete remission; the only remaining FDG uptake is seen in the kidneys (b, right panel). Imaging of lung metastases. In patient 12, bilateral lung metastases

\section{Retransfusion and Toxicity}

On average, 71 million clonally expanded autologous tumor-reactive T-lymphocytes were retransfused to each patient (Table 1). No toxic side-effects, such as fever, chills, malaise, severe fluid retention, pulmonary edema or respiratory distress, were observed.

\section{Clinical Outcome}

The patients were followed for 36 months on average (range 6-51 months) and monitored in accordance with the Swedish colorectal cancer follow-up protocol. The patients with distant metastases (stage IV) responded to treatment, with either extended periods of stable disease $(n=4)$, partial response with diminished tumor burden $(n=1)$ or complete response with no detectable remaining tumor $(n=4)$ (Table 1). Patient no. 14 (Table 1) with disseminated disease had a large sigmoid tumor resected en bloc, involving the left ovary, the left uterine tube, and the upper part of the uterus. She had metastases in both liver lobes. The metastases regressed completely after immunotherapy, as demonstrated by CT scan (Fig. 3a) and FDG-PET (Fig. 3b). Patient no. 12 (Table 1) displayed disseminated disease with large liver metastases and multiple lung metastases. The primary tumor was resected because of

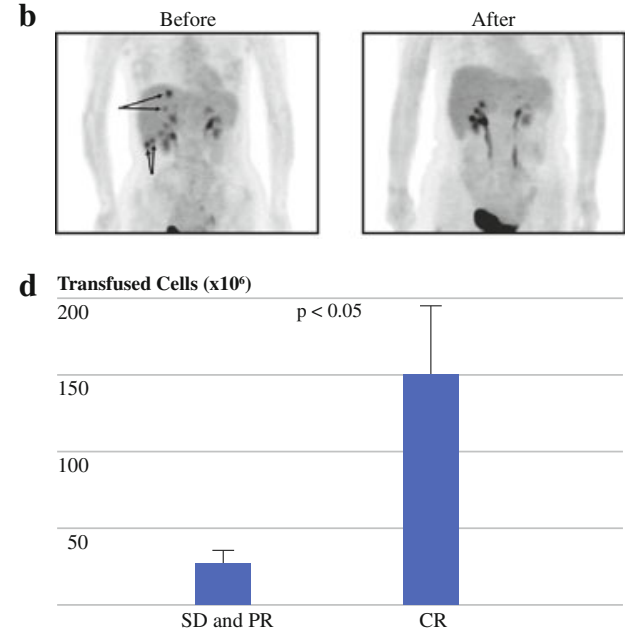

were detected on computer tomography (CT) prior to surgery (c, left panel). Follow-up CT scan 3 months after cell transfusion revealed complete remission of some metastases and significant decrease in the remaining metastases (c, right panel). Dose-dependent response in the stage IV patients (d). Complete response was seen in four of the nine stage IV patients. Patients with complete response were transfused with an average of 150 million cells, a significantly $(P<0.05)$ larger number of cells than the average of 27 million cells transfused in patients with partial response or stable disease

obstructive symptoms, and immunotherapy followed after 5 weeks. Partial response with marked regression of liver metastases and disappearance of several lung metastases occurred within 3 months (Fig. 3c). Notably, the stage IV patients responding with SD and PR received significantly fewer cells than the four patients with $\mathrm{CR}(P<0.05)$ (Fig. 3d), thus indicating a dose response.

The cumulative survival of the nine treated stage IV patients was compared with all stage IV cases in the Stockholm region during the year of $2003(n=174)$. The median survival of stage IV patients who received immunotherapy was 2.6 years compared with 0.8 years median survival of the control group, and there was a tendency towards increased cumulative survival $(P=0.06)$ (Fig. 4e). Six patients have died, of whom five were classified as responders with stable disease (nos. 8 and 11), partial response (no. 12) or complete response (nos. 13 and 14). Patient 9 was lost to follow-up. Patients no. 13 and 14 were classified as complete responders after immunotherapy. However, patient 14 developed metastases to the liver after 3 years and died after 40 months. Patient no. 13 died from pancreatic cancer without any signs of recurrent colon cancer. Details regarding dead patients are presented in Table 1 .

Five patients classified as stage II, with high-risk tumor characteristics, were included in the study (Table 1). One stage II patient (no. 2) developed liver recurrences, which 
a

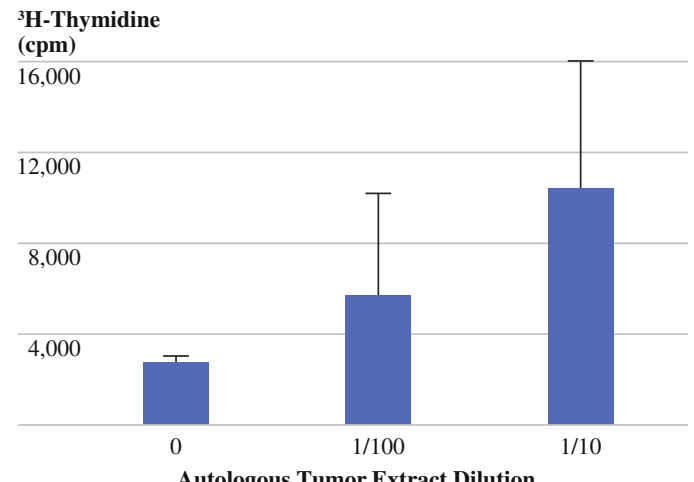

c ${ }^{3}$ H-Thymidine

(cpm)

12,000

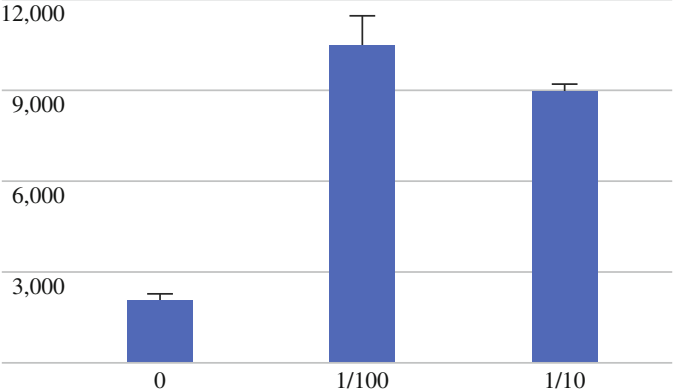

Autologous Tumor Extract Dilution

e

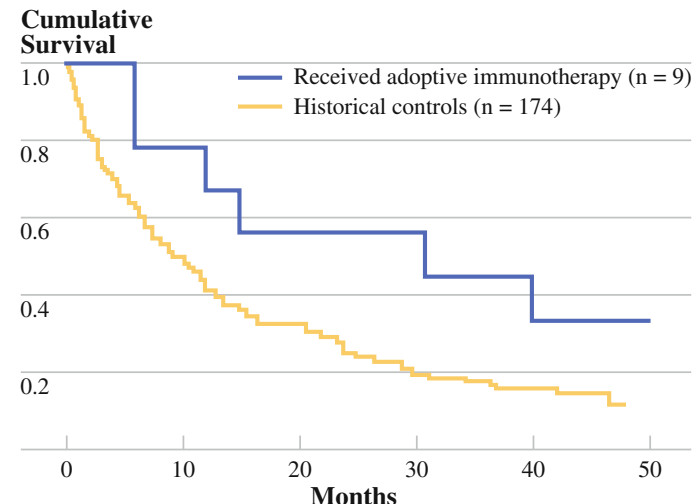

FIG. 4 Treatment responses after sentinel-node-based adoptive immunotherapy. Immune response upon stimulation with autologous tumor extract could be detected in peripheral blood leukocytes from patient 10 (a and b). Patient 10 had, 10 months earlier, received a transfusion of ex vivo expanded sentinel node lymphocytes. The proliferative response (a) was measured by ${ }^{3} \mathrm{H}$-thymidine incorporation assay (day 5), and the amount of IFN- $\gamma$ secreted into the supernatant was measured by ELISA (day 6) (b). Stimulation with autologous tumor extract diluted $1 / 100$ or $1 / 10$ or in medium alone (0). In patient 16 the proliferative response in peripheral blood after transfusion, at 10 months (c) and 42 months (d), was investigated by ${ }^{3} \mathrm{H}$-thymidine incorporation assay. At 10 months, peak proliferation

responded to immunotherapy with regression of metastases. $\mathrm{He}$ is now free of recurrences and classified as having stable disease. The other patients with high-risk stage II disease b IFN- $\gamma$

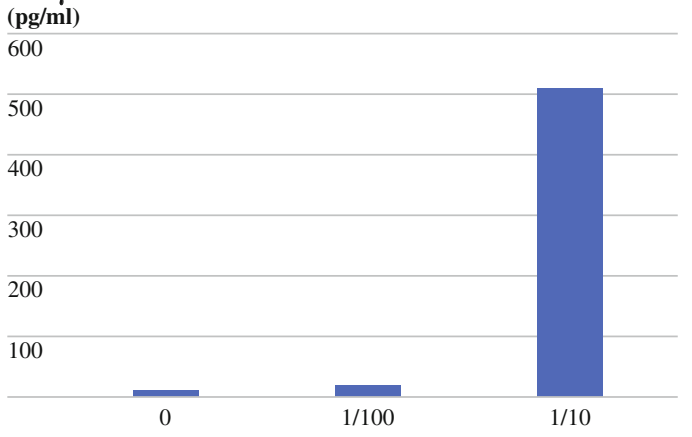

Autologous Tumor Extract Dilution

d ${ }^{3}$ H-Thymidine

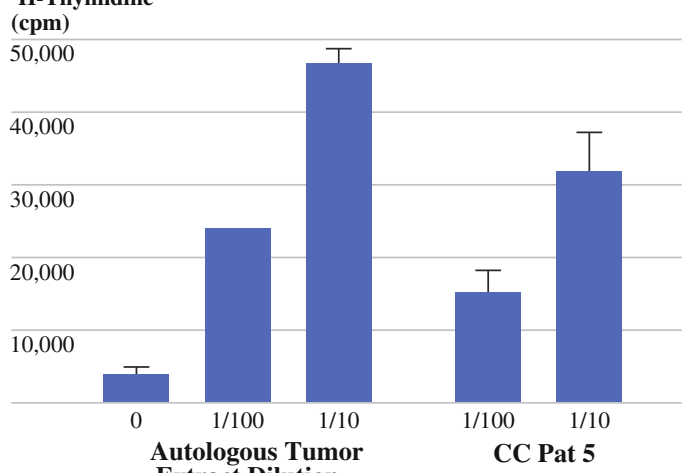

Extract Dilution

CC Pat 5

upon stimulation with autologous tumor homogenate (diluted 1/100 and 1/10) was approximately 10,000 cpm (day 7) (c). By 42 months the peak proliferation had increased to $47,000 \mathrm{cpm}$ (day 6) upon addition of autologous tumor homogenate (diluted 1/100 and 1/10) (d). Proliferation in response to addition of allogeneic tumor homogenate from patient 5 was also investigated ("CC pat 5 1/ $100 "$ and "CC pat $51 / 10 ")$, resulting in peak proliferation of $31,000 \mathrm{cpm}$ (day 6) (d). Increased survival in stage IV patients treated with sentinel-node-based adoptive immunotherapy (e). Actuarial survival curves were obtained by comparing survival among patients with stage IV receiving adoptive immunotherapy ( 9 patients) versus historical controls diagnosed in the year 2003 (174 cases)

(nos. 1 and 3-5) and the patients with stage III (nos. 6 and 7) have had no signs of recurrences and are thus classified as stable disease according to their original status. 


\section{Posttreatment Immunological Evaluation}

We tested peripheral blood leukocytes for their memory capacity by proliferation assays in two patients (nos. 10 and 16 ) by recalling reactivity against autologous tumor extract. Ten months after transfusion we detected proliferation and secretion of IFN- $\gamma$ in response to autologous tumor extract (Fig. 4a-c), indicating the presence of memory cells. Peripheral blood leukocytes from patient no. 16 were again tested for proliferation against autologous tumor extract 42 months after treatment. Interestingly an increased recall proliferation was noticed after 42 months $(47,000 \mathrm{cpm})$ as compared with responses seen after 10 months $(10,000 \mathrm{cpm})$ (Fig. 4c, d), suggesting that expansion of a long-lived memory cell population occurred in vivo. When these cells were tested for proliferation against allogeneic tumor extract from patient 5, a recall response was noted, albeit at a lower level, suggesting that common tumor antigens are present in these tumor preparations (Fig. 4d).

\section{DISCUSSION}

We have previously identified the localized presence of immune responses against the autologous tumor in sentinel nodes draining colorectal and urinary bladder cancers. ${ }^{12-14}$ In the present study, sentinel-node-acquired lymphocytes were expanded ex vivo against an autologous tumor extract and retransfused to 16 patients with colorectal cancer.

In theory, processing and presentation of exogenous intact antigens via endocytosis favors loading of the major histocompatibility complex (MHC) class II pocket, thus mediating clonal expansion of class II restricted tumorreactive $\mathrm{CD}^{+}{ }^{+} \mathrm{T}$-helper lymphocytes. This also appeared to be the case, as evidenced by an increased CD4/CD8 ratio in the majority of cell cultures (Table 1). However, in some cases a significant expansion of $\mathrm{CD}^{+}$cytotoxic T-cells was observed, most likely the result of cross-presentation of extracellular antigens on MHC class I molecules. ${ }^{25}$ The expanded cell populations displayed the surface phenotype of memory cells (Fig. 2a) and a cytokine secretion profile of Th1-cells (Fig. 2c). Both central (CD62L-positive) and effector (CD62L-negative) cells were present in the transfused cell populations (Fig. 2a). Whereas effector memory cells are capable of executing immediate effector functions upon antigen encounter, central memory cells home to lymph nodes and may provide a lifelong source of new effector cells, both upon secondary stimulation and under the influence of homeostatic cytokines. ${ }^{26,27}$ Indeed, both proliferation and IFN- $\gamma$ secretion in response to tumor extract could be detected in peripheral blood 10-42 months after treatment (Fig. 4a-d).
On average, 71 million clonally expanded lymphocytes were used in the adoptive transfers, which is a relatively small number compared with protocols using primarily $\mathrm{CD}^{+}$cytotoxic $^{\text {T-cells. }}{ }^{28} \mathrm{We}$ consider this justifiable, since as few as $3 \times 10^{5}$ adoptively transferred, antigenspecific $\mathrm{CD}^{+}$Th1-cells are able to mediate tumor regression in mice, mainly through recruitment and expansion of host $\mathrm{CD}^{+}{ }^{+}$T-cells. $^{29}$ Indeed, four patients with distant metastases (stage IV) displayed complete tumor regression after treatment with sentinel-nodeacquired lymphocytes. However, the five stage IV patients with PR or SD all received lymphocytes in the lower range (Fig. 3a), suggesting overwhelming immunosuppression from their residual metastatic disease. Even when an antitumoral response is induced by transfer of tumorreactive $\mathrm{CD} 4^{+}$Th1-lymphocytes, tumor cells may escape elimination by losing targeted antigens, rendering T-cells anergic by downregulation of costimulatory molecules, by inducing regulatory T-lymphocytes, or by specifically deleting responding T-lymphocytes. ${ }^{30-33}$ The tumor that finally develops may reflect a selection of poorly immunogenic and/or immune-resistant malignant cells. ${ }^{34}$ Tumorinduced immunomodulation may not be limited to the tumor microenvironment. Indeed, immunosuppression has been identified also in lymphocytes from sentinel nodes, which has been suggested to facilitate lymph node metastases. ${ }^{35}$ However, our method appears to reconstitute the function of tumor-reactive $\mathrm{CD} 4^{+}$Th1-lymphocytes and provide a large number of functioning tumor-reactive $\mathrm{T}$ lymphocytes, resulting in large antitumoral responses in patients receiving large numbers of tumor-reactive T-cells. Thus, an iterated therapy may be advocated, especially in the presence of a significant tumor burden.

Life expectancy in stage IV patients is $8-12$ months. ${ }^{36}$ Even though the cumulative survival was not significantly different from in conventionally treated controls, likely due to the limited number of patients included in our study, the median survival of 2.6 years in the nine immunotherapytreated stage IV patients is encouraging (Fig. 4e). The possibility of receiving immunotherapy tended to select patients hesitant to subject themselves to chemotherapy, and therefore the overall amount of chemotherapy used was low. Most patients received only immunotherapy (as described in the "Methods" section). Among the four complete responders, two patients (nos. 14 and 16) received chemotherapy in association with immunotherapy, whereas the other two patients had chemotherapy over 1 year prior to immunotherapy (patients no. 13 and 15 ). Thus, the clinical results among immunotherapy-treated stage IV patients are unlikely due to chemotherapy. Theoretically, chemotherapy is a two-edged sword. Chemotherapy may decrease the proliferative capability of tumor-reactive T-cells since chemotherapy affects dividing 
cells, and therefore results may have been underestimated. On the other hand, when chemotherapy is administered, the increased tumor cell death will result in increased antigen presentation to T-lymphocytes, thus increased $\mathrm{T}$-cell reactivity may occur, as discussed in a recent review. ${ }^{37} \mathrm{It}$ would be interesting to test immunotherapy and chemotherapy in combination in a future systematic study.

To our knowledge, sentinel-node-based immunotherapy in patients with colon cancer is a novel approach. There are some major differences between our study and others that use anti-CD3/anti-CD28 expanded tumor-infiltrating lymphocytes, myeloablation, and high-dose IL-2. ${ }^{28}$ Firstly, we use sentinel-node-acquired lymphocytes providing a natural antitumoral response, enhanced by ex vivo stimulation with autologous tumor homogenate, physiological doses of IL-2, and restimulation with autologous antigen-presenting cells (APCs) on one occasion after about 3 weeks of expansion. Only T-lymphocytes with high affinity to antigens from the primary tumor will survive until transfusion. Secondly, myeloablation and high-dose IL-2 regimen entail high toxicity and bring along risk of severe complications, long treatment periods, and high costs. Our transfusions, however, were given without complications, during a period of $1 \mathrm{~h}$, with patients often discharged the same day. Thirdly, our protocol aims at expansion of $\mathrm{CD}^{+}$Th1lymphocytes from sentinel nodes, as opposed to expansion of cytotoxic $\mathrm{CD}^{+}$T-lymphocytes harvested as tumorinfiltrating lymphocytes.

In a recent retrospective analysis of resected tumors and lymph nodes from patients with colorectal cancer, the influence of immune cells on the advancement and outcome of disease was investigated. ${ }^{24}$ Increased numbers of memory effector cells, Th1 activation as indicated by increased messenger RNA (mRNA) for IFN $-\gamma$, as well as presence of the Th1-inducing transcription factor T-bet within the tumor were all associated with absence of early metastatic invasion and correlated strongly with prolonged survival. Notably, the patients in our study were treated with the same population of Th1-lymphocytes. Interestingly, a recent experimental mouse model supports the efficacy of $\mathrm{CD}^{+}$T-helper lymphocytes in adoptive immunotherapy. Mice with established tumors were treated with adoptive transfer using in vitro activated T-lymphocytes harvested from tumor-draining lymph nodes. The results demonstrated that expanded sorted $\mathrm{CD} 4^{+} \mathrm{T}$-helper lymphocytes from the tumor-draining lymph node have therapeutic efficacy on their own, and a synergistic effect was found when $\mathrm{CD} 4^{+} \mathrm{T}$-helper lymphocytes were used in combination with expanded cytotoxic $\mathrm{CD} 8^{+} \mathrm{T}$-lymphocytes. ${ }^{38}$ In addition, adoptive transfer of a tumor antigenspecific $\mathrm{CD} 4^{+} \mathrm{T}$-cell clone to one patient with metastatic malignant melanoma was recently reported to induce a 2month clinical remission. ${ }^{39}$ In the light of these findings, future trials using sentinel-node-acquired $\mathrm{CD} 4^{+}$and $\mathrm{CD} 8^{+}$ T-lymphocytes, raised separately under optimal conditions, may further improve the outcome of sentinel-node-based adaptive immunotherapy.

In conclusion, our study shows the novel finding that freshly isolated sentinel-node-acquired lymphocytes possess proliferative ability ex vivo against autologous tumor homogenate and may be retransfused to the patient without complications, as adoptive immunotherapy. Our results indicate that this cell population is capable of inducing tumor regression in patients with disseminated colorectal cancer. Due to its generic design, i.e., identification of sentinel node, isolation of tumor-reactive lymphocytes, and ex vivo expansion, this method may be used to treat other types of disseminated solid cancers.

ACKNOWLEDGMENT This work was supported by the Swedish Cancer Society, the Cancer and Allergy Society, the Hedlunds Foundation, the Lundberg Foundation, the Gustav V Foundation, the Söderberg Foundation, the Selander Foundation, the Wallenberg Foundation, and the Åke Wiberg Foundation. We are grateful for linguistic advice from Associate Professor Robert A. Harris.

OPEN ACCESS This article is distributed under the terms of the Creative Commons Attribution Noncommercial License which permits any noncommercial use, distribution, and reproduction in any medium, provided the original author(s) and source are credited.

\section{REFERENCES}

1. Fearly J. GLOBOCAN 2002. Descriptive Epidemiology Group, International Agency for Research on Cancer. Lyon, France.: www-dep.iarc.fr/; 2002. Accessed 21 Jan 2010.

2. Greenlee RT, Murray T, Bolden S, Wingo PA. Cancer statistics, 2000. CA Cancer J Clin. 2000;50:7-33.

3. O'Connell JB, Maggard MA, Ko CY. Colon cancer survival rates with the new American Joint Committee on Cancer sixth edition staging. J Natl Cancer Inst. 2004;96:1420-5.

4. Moertel CG, Fleming TR, Macdonald JS, et al. Levamisole and fluorouracil for adjuvant therapy of resected colon carcinoma. $N$ Engl J Med. 1990;322:352-8.

5. Moertel CG, Fleming TR, Macdonald JS, et al. Fluorouracil plus levamisole as effective adjuvant therapy after resection of stage III colon carcinoma: a final report. Ann Intern Med. 1995;122:321-6.

6. Quasar Collaborative G, Gray R, Barnwell J, et al. Adjuvant chemotherapy versus observation in patients with colorectal cancer: a randomised study. Lancet. 2007;370:2020-9.

7. Mayo C. Cancer of the large bowel. Med Sentinel. 1904; 12:46673.

8. Swanson RS, Compton CC, Stewart AK, Bland KI. The prognosis of T3N0 colon cancer is dependent on the number of lymph nodes examined. Ann Surg Oncol. 2003;10:65-71.

9. Cabanas RM. An approach for the treatment of penile carcinoma. Cancer. 1977;39:456-66.

10. Dahl K, Westlin J, Kraaz W, et al. Identification of sentinel nodes in patients with colon cancer. Eur J Surg Oncol. 2005;31:381-5.

11. Saha S, Dan AG, Beutler T, et al. Sentinel lymph node mapping technique in colon cancer. Semin Oncol. 2004;31:374-81.

12. Marits P, Karlsson M, Dahl K, et al. Sentinel node lymphocytes: tumour reactive lymphocytes identified intraoperatively for the 
use in immunotherapy of colon cancer. $\mathrm{Br} J$ Cancer. 2006;94:1478-84.

13. Marits P, Karlsson M, Sherif A, et al. Detection of immune responses against urinary bladder cancer in sentinel lymph nodes. Eur Urol. 2006;49:59-70.

14. Dahl K, Karlsson M, Marits P, et al. Metinel node-the first lymph node draining a metastasis-contains tumor-reactive lymphocytes. Ann Surg Oncol. 2008; 15:1454-63.

15. Hanna MG, Jr, Hoover HC, Jr, Vermorken JB, et al. Adjuvant active specific immunotherapy of stage II and stage III colon cancer with an autologous tumor cell vaccine: first randomized phase III trials show promise. Vaccine. 2001; 19:2576-82.

16. Mocellin S, Rossi CR, Lise M, Nitti D. Colorectal cancer vaccines: principles, results, and perspectives. Gastroenterology. 2004;127:1821-37.

17. Fong L, Hou Y, Rivas A, et al. Altered peptide ligand vaccination with Flt3 ligand expanded dendritic cells for tumor immunotherapy. Proc Natl Acad Sci USA. 2001;98:8809-14.

18. Dudley ME, Wunderlich JR, Yang JC, et al. Adoptive cell transfer therapy following non-myeloablative but lymphodepleting chemotherapy for the treatment of patients with refractory metastatic melanoma. J Clin Oncol. 2005;23:2346-57.

19. Morgan RA, Dudley ME, Wunderlich JR, et al. Cancer regression in patients after transfer of genetically engineered lymphocytes. Science. 2006;314:126-9.

20. Camacho SA, Heath WR, Carbone FR, et al. A key role for ICAM-1 in generating effector cells mediating inflammatory responses. Nature Immunol. 2001;2:523-9.

21. Antony PA, Piccirillo CA, Akpinarli A, et al. CD8+ T cell immunity against a tumor/self-antigen is augmented by $\mathrm{CD} 4+\mathrm{T}$ helper cells and hindered by naturally occurring $\mathrm{T}$ regulatory cells. J Immunol. 2005;174:2591-601.

22. Simmons WJ, Koneru M, Mohindru M, et al. Tim-3+ T-bet+ tumor-specific Th1 cells colocalize with and inhibit development and growth of murine neoplasms. J Immunol. 2005;174:1405-15.

23. Peng SL, Townsend MJ, Hecht JL, et al. T-bet regulates metastasis rate in a murine model of primary prostate cancer. Cancer Res. 2004;64:452-5.

24. Pages F, Berger A, Camus M, et al. Effector memory T cells, early metastasis, and survival in colorectal cancer. $N$ Engl $\mathrm{J}$ Med. 2005;353:2654-66.

25. Shen L, Rock KL. Priming of T cells by exogenous antigen crosspresented on MHC class I molecules. Curr Opin Immunol. 2006;18:85-91.
26. Geginat J, Sallusto F, Lanzavecchia A. Cytokine-driven proliferation and differentiation of human naive, central memory, and effector memory CD4(+) T cells. J Exp Med. 2001;194:1711-9.

27. Hammarlund E, Lewis MW, Hansen SG, et al. Duration of antiviral immunity after smallpox vaccination. Nat Med. 2003;9:1131-7.

28. Dudley M, Wunderlich J, Robbins P, et al. Cancer regression and autoimmunity in patients after clonal repopulation with antitumor lymphocytes. Science. 2002;298:850-4.

29. Surman DR, Dudley ME, Overwijk WW, Restifo NP. Cutting edge: $\mathrm{CD} 4+\mathrm{T}$ cell control of CD8 $+\mathrm{T}$ cell reactivity to a model tumor antigen. J Immunol. 2000;164:562-5.

30. Staveley-O'Carroll K, Sotomayor E, Montgomery J, et al. Induction of antigen-specific T cell anergy: an early event in the course of tumor progression. Proc Natl Acad Sci. 1998;95:1178-83.

31. Woo EY, Yeh H, Chu CS, et al. Cutting edge: regulatory T cells from lung cancer patients directly inhibit autologous $\mathrm{T}$ cell proliferation. J Immunol. 2002;168:4272-6.

32. Engelhard VH, Bullock TN, Colella TA, et al. Antigens derived from melanocyte differentiation proteins: self-tolerance, autoimmunity, and use for cancer immunotherapy. Immunol Rev. 2002;188:136-46.

33. Lee PP, Yee C, Savage PA, et al. Characterization of circulating $\mathrm{T}$ cells specific for tumor-associated antigens in melanoma patients. Nat Med. 1999;5:677-85.

34. Dunn GP, Old LJ, Schreiber RD. The three e's of cancer immunoediting. Ann Rev Immunol. 2004;22:329-60.

35. Cochran AJ, Huang RR, Lee J, et al. Tumour-induced immune modulation of sentinel lymph nodes. Nat Rev Immunol. 2006;6:659-70.

36. Best L, Simmonds P, Baughan C, Buchanan R, Davis C, Fentiman I, et al. Palliative chemotherapy for advanced or metastatic colorectal cancer. Colorectal Meta-analysis Collaboration. Cochrane Database Syst Rev. 2000:CD001545.

37. Zitvogel L, Apetoh L, Ghiringhelli F, Kroemer G. Immunological aspects of cancer chemotherapy. Nat Rev Immunol. 2008;8:5973.

38. Wang LX, Shu S, Disis ML, Plautz GE. Adoptive transfer of tumor-primed, in vitro-activated, CD4 $+\mathrm{T}$ effector cells (TEs) combined with CD8 + TEs provides intratumoral TE proliferation and synergistic antitumor response. Blood. 2007;109:4865-76.

39. Hunder NN, Wallen H, Cao J, et al. Treatment of metastatic melanoma with autologous CD4+ T cells against NY-ESO-1. $N$ Engl J Med. 2008;358:2698-703. 